\title{
MedienPädagogik
}

Zeitschrift für Theorie und Praxis der Medienbildung

\section{Editorial: Offenheit im Kontext von Medien- und Hochschulentwicklung}

\author{
Beiträge und Impulse des wissenschaftlichen Nachwuchses aus \\ (Lehr-)Praxis und Forschung
}

Franziska Bellinger und Anna Heudorfer

Das Junge Forum für Medien und Hochschulentwicklung (JFMH)

Das vorliegende Heft 32 der Zeitschrift MedienPädagogik steht unter dem Thema «Offenheit in Lehre und Forschung - Königsweg oder Sackgasse?». Es fungiert zugleich als Tagungsband zum Jungen Forum für Medien und Hochschulentwicklung, das vom 15. bis 16. Juni 2017 am Hamburger Zentrum für Universitäres Lehren und Lernen (HUL) der Universität Hamburg stattfand (www.jfmh2017.uni-hamburg.de).

Das Junge Forum für Medien und Hochschulentwicklung (JFMH) findet seit 2012 jährlich statt und versteht sich als Nachwuchstagung unter der Schirmherrschaft der Deutschen Gesellschaft für Hochschuldidaktik (dghd), der Gesellschaft für Medien in der Wissenschaft (GMW), der Gesellschaft für Informatik (GI; Fachgruppe E-Learning) sowie der Deutschen Gesellschaft für Erziehungswissenschaft (DGfE; Sektion Medienpädagogik).

Als ein Forum des Austausches richtet sich das JFMH einerseits an Nachwuchswissenschaftlerinnen und Nachwuchswissenschaftler in der Qualifizierungsphase (Young Researchers) sowie andererseits an junge Wissenschaftlerinnen und Wissenschaftler aus praxisorientierten Entwicklungs- und Innovationsprojekten (Young Professionals), die am Anfang ihrer beruflichen Tätigkeit im Feld Medien und Hochschulentwicklung stehen. Das Forum steht Interessierten aus den Feldern Hochschuldidaktik, Mediendidaktik, Medienpädagogik und E-Learning offen und versucht den Austausch über die einzelnen Themenbereiche hinweg zu ermöglichen.

Das JFMH hat folglich zum Ziel, den interdisziplinären, einrichtungs- und funktionsübergreifenden Diskurs zu stärken. Durch die Beteiligung von Young Professionals und Young Researchers wird ein ausgewogener Theorie-Praxis-Dialog angestrebt. Erfahrene Wissenschaftlerinnen und Wissenschaftler verschiedener Disziplinen sind zudem eingeladen, die Beiträge aus verschiedenen Perspektiven zu kommentieren sowie die Nachwuchswissenschaftlerinnen und Nachwuchswissenschaftler zu unterstützen und zu begleiten. Austausch, Vernetzung und produktives Feedback stehen im Vordergrund, um die Teilnehmerinnen und Teilnehmer in frühen Phasen des wissenschaftlichen Arbeitens und Publizierens bestmöglich zu fördern. 
Über die letzten Jahre hat sich das JFMH als Veranstaltung etabliert, auf der Nachwuchswissenschaftlerinnen und Nachwuchswissenschaftler, die erste Schritte im Wissenschaftssystem wagen, auf (beruflich) fortgeschrittene Wissenschaftlerinnen und Wissenschaftler treffen, welche die Tagung seit Jahren gerne besuchen und aktiv daran partizipieren. So kommen Interessentinnen und Interessenten unterschiedlicher Erfahrungs- und Qualifikationsstufen miteinander ins Gespräch, beraten sich gegenseitig in ihrer Arbeit und entwickeln gemeinsam neue Ideen. Der Austausch auf Augenhöhe und eine Atmosphäre, die (möglichst) frei von Leistungsdenken und -druck ist, zeichnen das JFMH aus. Im vorliegenden Themenheft zeigt sich daher deutlich, dass das Thema "Offenheit» nicht nur inhaltlich, sondern auch konzeptionell vom wissenschaftlichen Nachwuchs umgesetzt wird.

Während der zwei Konferenztage im Juni 2017 haben wir neben eher klassischen Tagungsformaten - wie Vorträgen und Workshops - zwei Sessions umgesetzt, die sich nicht den Inhalten der Tagung, sondern der Weiterentwicklung des JFMH selbst widmeten. Ziel war es, die Teilnehmerinnen und Teilnehmer verstärkt in die Planung und kreative Fortschreibung des Jungen Forums einzubinden und hierfür gemeinsam neue Ideen zu entwickeln. Anlässlich des fünfjährigen Jubiläums konnten wir die Initiatorinnen des JFMHs (Miriam Barnat, Diana Bücker, Sandra Hofhues, Cornelia Kenneweg und Marianne Merkt) für eine Podiumsdiskussion gewinnen. Sie blickten auf die letzten Jahre zurück, erinnerten dabei die Anfänge, zeichneten die Entwicklung des JFMH nach und teilten ihre persönlichen Höhepunkte sowie Zukunftsgedanken mit dem Publikum (online auf der Tagungswebsite als Podcast verfügbar). Im Anschluss an den «Rückblick und Ausblick nach fünf Jahren JFMH» wagten wir mit einer Zukunftswerkstatt unter dem Motto «Forum 7: Update \& Remake», moderiert von Jan Vanvinkenroye und Angelika Thielsch, den Blick nach vorne. In einem gut besuchten Slot wurden Utopien einer idealen Tagung kollaborativ in umsetzbare Vorschläge für nachfolgende Junge Foren für Medien und Hochschulentwicklung verwandelt.

\section{Zum Thema des JFMH 17: Offenheit in Lehre und Forschung - Königsweg oder Sack- gasse?}

Das Tagungsthema des JFMH 2017 schloss an den Diskurs um die Digitalisierung an Hochschulen und damit einhergehenden Strategien formaler Bildungsinstitutionen an: Die Idee einer Open Education unterstützt durch offene Lernmaterialien und Bildungsressourcen (Open Educational Resources) sowie dadurch entstehende Praktiken (Open Educational Practices) sollten im Mittelpunkt der zwei Konferenztage stehen. Dabei war es uns besonders wichtig, das Konzept von «Offenheit» für das JFMH 2017 weit zu fassen, um Themen wie Open Access als Form der digitalen Veröffentlichungspraxis in der Forschung berücksichtigten zu können. Ziel des Jungen Forums 2017 an der Universität Hamburg war es, Potenziale und Grenzen offener Bildungspraktiken in allen Bildungsbereichen und schwerpunktmässig an Hochschulen 
kritisch zu diskutieren sowie aus verschiedenen Perspektiven zu beleuchten. Um die Breite des Themas schon im Call for Participation aufzuzeigen, eröffneten wir fünf Ebenen, die in den Vorträgen und Workshops der Tagung auf unterschiedliche Art und Weise aufgegriffen wurden:

a. Die didaktisch-methodische-Ebene fokussierte Fragen rund um die Gestaltung akademischer Lehre unter der Idee einer Open Education: Wie können sich Open Educational Practices (OEP) konstituieren und in die akademische Lehre integriert werden? Welche (medien-)didaktischen Handlungsspielräume eröffnen sich durch die Gestaltung offener Lehr-/Lernszenarien? Wo liegen Hürden oder gar Grenzen? Welche Rolle nehmen in diesem Zusammenhang die Bildungsorganisationen, aber auch Lehrende sowie Lernende ein und mit welchen Aufgaben und Herausforderungen sehen sich die Akteurinnen und Akteure jeweils konfrontiert? Welche neuen Zielgruppen lassen sich für Forschung und Lehre erreichen?

b. Die theoretisch-begriffliche-Ebene stellte die Auseinandersetzung mit Termini im Kontext «Open Education» in den Vordergrund und fragte nach der Bedeutung sowie Auslegung von Offenheit im (akademischen) Diskurs: Was bedeutet Offenheit für Hochschulen und weitere (formale) Bildungsinstitutionen? Wie wurde der Begriff in den vergangenen Jahren besetzt und wie gelingt es, sich ggfs. davon zu lösen? Woher rühren Konzepte wie Open Educational Ressources (OER), Open Educational Practice (OEP), Open Science (OS) sowie Open Access (OA) und welche bildungstheoretischen, aber auch philosophischen Annahmen liegen dahinter? Wie lassen sich dahinter liegende Annahmen offenlegen und reflektieren?

c. Die Ebene der Qualitätssicherung beleuchtete Fragen nach der Sicherung wissenschaftlichen Wissens vor dem Hintergrund der Bestrebung einer Open Education: Wie lässt sich Qualität unter der Leitidee «Offenheit» nachhaltig sichern? Welche Qualitätsmanagementverfahren haben sich bereits etabliert und als anschlussfähig erwiesen (bspw. Peer-Review-Verfahren)? Wo bedarf es neuer Überlegungen, um die Qualität von Forschung und akademischem Wissen zu sichern?

d. Die technische Ebene fokussierte Fragen nach digitalen Infrastrukturen, um «Offenheit» in Bildungsorganisationen zu ermöglichen: Inwieweit stellt die Idee der Offenen Bildung Hochschulen und weitere Bildungsinstitutionen vor technische Herausforderungen? Welche technischen Voraussetzungen (Formate, Protokolle) sind einzuhalten und wie lässt sich der Kulturwandel hin zur Offenheit technisch gestalten?

e. Die juristische Ebene rückte die Auseinandersetzung mit rechtlichen Herausforderungen ins Zentrum und ist eng verwoben mit den Ebenen $C$ und D: Welche juristischen Herausforderungen für Hochschulen und weitere Bildungsinstitutionen gehen mit der Idee der Offenen Bildung einher? Welche Lizensierungsmodelle (bspw. Creative Commons) werden bereits in Bildungsinstitutionen erprobt und welche Erfahrungswerte gibt es? Wie kann die Bestrebung zur Offenheit juristisch begleitet werden? 
Die Beiträge in diesem Themenheft

Wie können Studierende dazu beitragen, die Verbreitung von OER an der Hochschule zu fördern? Mit dieser Frage setzen sich Kathrin Braungardt, Matthias Kostrzewa und Christine Ruthenfranz am Beispiel der Ruhr-Universität Bochum (RUB) auseinander. Das dort ansässige Projekt der eScouts-Teams und die dazugehörige Plattform Open$R \cup B$ werden vorgestellt und kritisch reflektiert.

Bence Lukács und Constanze Reder widmen sich der Reflexion als Teil akademischer Medienkompetenz. Dabei wird zunächst ein Projekt beschrieben, in dem Studierende mit OER und OEP in Kontakt kommen. Im Anschluss wird die Nutzung eines projektbegleitenden Podcasts thematisiert, der zur Dokumentation und Reflexion eingesetzt wurde. Lukács und Reder erörtern Potenziale dieses Vorgehens für den reflektierten Umgang einer offenen Lehr-Lernpraxis an der Hochschule.

Offene Online-Kurse (MOOCs) sind an Hochschulen im OER-Format zur akademischen Weiterbildung nutzbar. Ein Beispiel der Fachhochschule Lübeck stellt Anja Lorenz mit dem Projekt pMOOCs vor, das sich gezielt an Berufstätige richtet. Das Projekt wird anhand von Nutzungszahlen und Evaluationsergebnissen beleuchtet, die Unterschiede im Nutzungsverhalten im Vergleich zu traditionellen Studierenden vermuten lassen.

Oliver Tacke fragt nach der technischen Infrastruktur, die im Kontext von OER verwendet wird. Sein Plädoyer für Open Source Software fusst auf den gemeinsamen Werten, die die Open Source- mit der OER-Bewegung teilt und der Notwendigkeit, die technische Infrastruktur nicht als reines Werkzeug zu betrachten. Am Beispiel der Software H5P zeigt der Autor, wie sich offene Software für OER nutzen lässt.

Die Qualität von OER rückt in Jane Brückners Beitrag in den Fokus. Sie betrachtet die aktuelle Diskussion um Qualitätskriterien und -nachweise für OER. Entgegen der Bestrebungen verschiedener Akteure, formalisierte Qualitätsstandards zu entwickeln, möchte sie die Nutzerinnen und Nutzer wieder in den Mittelpunkt stellen und den aktuellen Qualitätsbegriff (sowie sein Zustandekommen) kritisch hinterfragen.

Christopher Könitz untersucht in seinem Beitrag, inwieweit OER in eine «digitale Unmündigkeit» führen können. Hierfür setzt er sich mit dem OER-Begriff aus einer bildungstheoretisch-medienpädagogischen Perspektive auseinander, um Unschärfen, die mit dem Begriff verbunden sind, zu beleuchten und ein starkes "Copyleft» einzufordern. Ziel ist die Theoretisierung des OER-Begriffs vor dem Hintergrund von Medienkompetenz- und Medienbildungsmodellen.

OEP ist kein für sich allein stehendes Konzept, sondern weist zahlreiche Verbindungen zu anderen Bildungsansätzen auf. Tamara Heck und Anna Heudorfer betrachten Parallelen und Integrationsmöglichkeiten von OEP und forschendem Lernen. Konzepte wie Open Science stellen dabei eine Brücke zwischen OEP und der wissenschaftlichen Ausbildung der Studierenden dar. Daran zeigen die Autorinnen, inwieweit sich Aspekte offener Lehr-/Lernpraktiken in das Konzept des forschenden Lernens integrieren lassen. 
Ricarda Bolten bewegt sich im Feld der Erwachsenenbildung und stellt die Frage, von welchen Faktoren die individuelle medienpädagogische Professionalisierung von Lehrenden in diesem Bereich abhängt. Dabei nutzt sie das Konzept des medialen Habitus und diskutiert Anreize, die die Entscheidungen Lehrender zur medienpädagogischen Professionalisierung beeinflussen.

Franziska Bellinger, Patrick Bettinger und Valentin Dander gehen vom Begriff der offenen Bildungspraktiken (OEP) aus und kritisieren, dass dieser weder theoretisch noch empirisch ausreichend fundiert ist. Sie schlagen einen praxeologisch-diskursanalytischen Forschungszugang vor, um OEP an den praxistheoretischen Diskurs anschlussfähig zu machen und sich einer Definition anzunähern.

Das Feld Open Science-Forschung wird abschliessend im Beitrag von Isabel Steinhardt diskutiert. Die Autorin reflektiert ein qualitativ-ausgerichtetes Forschungsprojekt anhand von fünf Ebenen und antizipiert Hürden und Grenzen, welchen Forscherinnen und Forscher im Kontext Open Science begegnen können.

\section{Danksagung}

Abschliessend möchten wir unseren Dank an diejenigen aussprechen, ohne die weder die Tagung, noch dieses Themenheft möglich gewesen wären. An erster Stelle danken wir den Autorinnen und Autoren dieses Themenhefts sowie den Referentinnen und Referenten, Podiumsdiskutantinnen sowie Moderatorinnen und Moderatoren des JFMH 2017. Ausserdem danken wir den Mentorinnen und Mentoren, die die Beitragenden intensiv unterstützt und im Publikationsprozess begleitet haben. Das JFMH zeichnet sich durch einen Mentoring-Prozess aus, der weit über klassische Begutachtungsverfahren hinausgeht. Wir haben uns sehr über die Unterstützung von erfahreneren Wissenschaftlerinnen und Wissenschaftlern gefreut, die dem wissenschaftlichen Nachwuchs bei der Erstellung und Verfeinerung ihrer Beiträge mit konstruktivem Feedback zur Seite standen.

Als nächstes danken wir der ausrichtenden Institution, dem Hamburger Zentrum für Universitäres Lehren und Lernen (HUL) der Universität Hamburg und vor allem der Leitung Prof. Dr. Gabi Reinmann sowie der stellvertretenden Leitung Prof. Dr. Kerstin Mayrberger für das Vertrauen und die Offenheit, die Tagung ausrichten sowie nach unserem Ermessen gestalten zu dürfen. Besonderer Dank gebührt unseren studentischen Hilfskräften, ohne die das Junge Forum nicht in dieser Form gelungen wäre: Herzlichen Dank an Amelie Nickel, Cansu Kartoglu, Marie Holtmann, Dragana Naskovska, Nataliia Zabolotna und Christoph Baldauf für die tatkräftige Unterstützung während der zwei Konferenztage und darüber hinaus. Daneben bedanken wir uns recht herzlich bei Marlen Beckmann für das Grafikdesign sowie bei Patrick Eickhoff, Carsten Hacker, Thorben Stender, Sofia Zicari und Ben Petzold für den technischen Support vor Ort. 
Nicht zuletzt sind die einzelnen Fachgesellschaften - die dghd, die GMW, die ELearning-AG der GI sowie die Sektion Medienpädagogik der DGfE und ihre jeweiligen Vertreterinnen und Vertreter - zu nennen. Wir danken für die finanzielle Unterstützung, ohne die wir das die Tagung nicht in der Form hätten veranstalten können. Vor allem waren aber die gemeinsamen Runden mit den Sprecherinnen und Sprechern der jeweiligen Nachwuchsgruppen immer wieder ein Gewinn und haben uns in der Planung der Tagung immer wieder inspiriert und vorangebracht.

Schliesslich möchten wir uns bei den Herausgeberinnen und Herausgebern der Zeitschrift MedienPädagogik für die Aufnahme unseres Tagungsbandes in das Themenheft 32 bedanken. In erster Linie gilt unser Dank Klaus Rummler, der uns technisch und inhaltlich bei der Erstellung des Heftes stets zur Seite stand. Auch Timo van Treeck, der die technische Umsetzung des Begutachtungsverfahrens im OnlineRedaktions-System mit uns testete, sei an dieser Stelle nicht vergessen.

Zuletzt wünschen wir allen Leserinnen und Lesern eine aufschlussreiche und unterhaltsame Lektüre.

Die Herausgeberinnen

Hamburg, Oktober 2018 